\title{
Mecanismo de Proteção em SDM-EON Ciente da Prioridade de Tráfego.
}

\author{
Rafael S. Lopes ${ }^{1}$, Denis Rosário ${ }^{1}$, Eduardo Cerqueira ${ }^{1}$, Helder Oliveira $^{1}$ \\ ${ }^{1}$ Universidade Federal do Pará (UFPA) - Belém, PA, Brasil \\ rafael.lopes@itec.ufpa.br \\ \{denis, cerqueira, heldermay\}aufpa.br
}

\begin{abstract}
With the increase in the number of devices connected to the Internet, caused mainly by the rise of the Internet of Things paradigm, the core of the network tends to suffer from the scarcity of resources, which, in turn, causes a higher connection blocking rate. This article proposes an algorithm for routing, spectrum and core allocation for space-division multiplexing elastic optical networks that use classes of service with different quality of protection levels to increase the acceptance rate of requests, guaranteeing $100 \%$ protection against failures to higher priority flows. The results demonstrate the proposed algorithm effectiveness in establishing connections with high priority compared to other algorithms found in the literature.
\end{abstract}

Resumo. Com o aumento do número de dispositivos conectados à Internet, ocasionado principalmente pela ascensão do paradigma de Internet das Coisas, o núcleo da rede tende a sofrer com a escassez de recursos, o que, por sua vez, causa maior taxa de bloqueio de conexões. Este artigo propõe um algoritmo de roteamento, alocação de espectro e núcleo para redes ópticas elásticas com multiplexação por divisão espacial que utiliza classes de serviço com diferentes níveis de qualidade de proteção para elevar o índice de aceitação de requisições, garantindo $100 \%$ de proteção contra falhas aos fluxos com prioridade mais alta. Os resultados demonstram a eficácia do algoritmo proposto no estabelecimento de conexões com prioridade alta em comparação com outros algoritmos presentes na literatura.

\section{Introdução}

O constante crescimento do número de usuários da Internet e a maior demanda por largura de banda em aplicações emergentes tem aproximado, cada vez mais, o núcleo da rede de seu limite físico de aprovisionamento de recursos. Dentre estas aplicações em expansão nos próximos anos, pode-se citar as conexões máquina a máquina ( $M 2 M-$ Machine-to-Machine), acessos através de smartphones e televisores como sendo as principais [Cisco 2020]. Sendo assim, percebe-se a necessidade de estudos que abordem novas formas para o transporte de dados, que suportem tal demanda, entregando qualidade de serviço ( $Q o S$ - Quality of Service) ao usuário final. Além da capacidade de transmissão, vale ressaltar a diversidade de requisitos dessas novas conexões. Por exemplo, serviços de mensagens instantâneas possuem maior exigência quanto à confiabilidade e vazão de dados, garantindo que a mensagem seja entregue sem perda de dados e no menor tempo possível. De outro modo, serviços de transmissão de vídeo em tempo real exigem maior largura de banda e menor latência, visando entregar melhor experiência ao usuário. 
Diante deste panorama emergem as redes ópticas elásticas (EON - Elastic Optical Networks), oferecendo melhor utilização do espectro através da maior granularidade em relação às redes ópticas com multiplexação por comprimento de onda (WDM - Wavelength Division Multiplexing). Nesta nova tecnologia o espectro passa a ser dividido em slots e não mais em canais, permitindo alocação flexível de acordo com a necessidade de cada fluxo [Boutaba et al. 2017]. No entanto, considerando a capacidade de comutação das fibras monomodo de núcleo único (comumente utilizadas nos backbones), presume-se que estas ainda serão insuficientes na provisão de recursos em um futuro próximo. Surgem ainda as redes ópticas elásticas com multiplexação por divisão espacial (SDM-EONS - Space-Division Multiplexing Elastic Optical Networks), aumentando as possibilidades de transporte de dados, através da utilização de fibras multinúcleo a capacidade de banda passante cresce a um fator $N$, sendo $N$ o número de núcleos em cada fibra.

O aumento da flexibilidade e disponibilidade de recursos, com o advento da tecnologia SDM-EON dá origem a novos problemas, que precisam ser solucionados para o melhor aproveitamento da rede. Um desses problemas é quanto ao roteamento e alocação de recursos. Diferente do que acontece nas redes $W D M$, em que rotas e comprimentos de onda são escolhidos para formar um caminho óptico, na SDM-EON é necessário encontrar a rota, o núcleo e a faixa de espectro. A faixa de espectro, por sua vez, é formada por um conjunto de slots de frequência que precisam ser contínuos e contíguos, o que aumenta a complexidade de encaminhamento e reserva de recursos nestas redes. Sendo assim, o que antes era o roteamento e alocação de comprimento de onda (RWA - Routing Wavelength Assignment) agora passa a ser chamado de roteamento e alocação de núcleo e espectro (RSCA - Routing, Spectrum and Core Allocation).

Com o incremento da quantidade de dados que podem ser transferidos simultaneamente através de $S D M-E O N$, falhas em enlaces e equipamentos podem causar ainda mais perdas que nas redes tradicionais. Portanto, há a necessidade de provisão de recursos durante tais ocorrências. As falhas em redes ópticas podem ser relacionadas aos equipamentos ou aos cortes de fibras, sendo esse último o mais comum. A taxa de falha de transmissores ópticos é de 10.867 FIT ${ }^{1}$, a de receptores é 4.311 FIT, e a de cortes de fibra, por ano, é de cerca 4 cortes para cada $1610 \mathrm{~km}$ de fibras [Zhang and Mukheriee 2004]. Os tempos médios de reparo para os equipamentos e cabos da rede são de 2 horas e 12 horas, respectivamente. De modo geral, existem duas abordagens que prevalecem na resiliência de redes: estratégias de proteção e de restauração.

Os métodos de proteção baseiam-se na alocação de recursos extras durante o roteamento do fluxo, garantindo rápida recuperação da conexão, mas com utilização ineficiente dos recursos da rede. Em contrapartida, técnicas de restauração se fundamentam no tratamento dinâmico, após a ocorrência da falha, permitindo assim a melhor utilização dos recursos, no entanto, exigindo novo cálculo de rotas e configuração dos comutadores, o que requer maior tempo de recuperação. Quanto à heterogeneidade do tráfego, cabe destacar os diferentes níveis de prioridade e requisitos. Por exemplo, aplicações de jogos em nuvem e telemedicina exigem maior largura de banda, baixa latência e baixa perda de pacotes [Laghari et al. 2019, De La Torre Díez et al. 2018]. Todavia, telemedicina exige maior relevância e garantia de que a conexão não será interrompida. Em uma situação de sobrecarga da rede, é aceitável que os esforços para o estabelecimento e sobrevivência dessas conexões não sejam iguais, pois é evidente o contraste entre as consequências que

\footnotetext{
${ }^{1} 1$ FIT $=1$ falha em 114.155 anos
} 
bloqueios de banda ou falhas no transporte de dados podem causar para ambos os fluxos.

Diante disso, percebe-se a importância de novos meios que garantam o atendimento de cada uma das requisições de acordo com suas necessidades, assegurando proteção mesmo em situações extremas, como as já citadas. O objetivo deste artigo é propor uma nova abordagem para o aprovisionamento de recursos em redes ópticas elásticas com multiplexação por divisão espacial, que garanta a qualidade de serviço e qualidade de proteção ( $Q o P$ - Quality of Protection), assumindo a economia de recursos da rede e a consequente diminuição das taxas de bloqueio de conexões. $\mathrm{O}$ algoritmo proposto considera a classificação dos fluxos por ordem de prioridade, utilizando graus de $Q o P$ e diferentes classes de serviço ( $\mathrm{CoSs}$ - Classes of Service), permitindo que, em caso de congestionamento da rede, a preferência seja para o estabelecimento de caminhos pelos fluxos com $C o S$ de maior $Q o P$.

A organização do restante do artigo foi feita da seguinte forma: na Seção 2 são apresentados alguns trabalhos relacionados e suas contribuições para a área sob estudo. A Seção 3 apresenta o algoritmo desenvolvido e seus principais aspectos. Na seção 4 é apresentada a ferramenta e os parâmetros utilizados para as simulações. Ainda na Seção 4, é explicada a metodologia e são expostos os resultados obtidos. Por fim, na Seção 5 está a conclusão do artigo.

\section{Trabalhos Relacionados}

As redes ópticas elásticas com multiplexação por divisão espacial têm impulsionado vários estudos para incrementar a alocação de recursos mantendo a proteção, no entanto, para o melhor do nosso conhecimento este é o primeiro trabalho que considerada diferentes prioridades nas requisições de tráfego dessas redes.

Os autores em [Santos et al. 2018] apresentam um modelo de tratamento de sobrecarga em redes ópticas elásticas que usa da degradação de serviço por $Q o S$ proporcional, considerando parâmetros de qualidade de diferenciação atribuídos pelos operadores da rede. O uso dessa técnica entrega menores taxas de bloqueio em situações de congestionamento da rede. No entanto, o algoritmo proposto não leva em conta a proteção aos fluxos, fazendo com que estes se percam caso ocorram falhas em suas rotas, além de não trabalhar com SDM-EONs. Foi apresentado em [Hai 2020] o conceito de proteção com reconhecimento de $Q o S$, que possibilita a separação dos fluxos em tráfegos de melhor esforço e tráfegos premium, fazendo com que, em caso de problemas na rede, apenas os tráfegos premium possuam proteção garantida, permitindo assim a recuperação rápida da conexão. No entanto, os autores não consideram $S D M-E O N$ e não utilizam uma política de liberação do espectro em benefício de fluxos com maior prioridade.

Os autores em [Hmaity et al. 2018] desenvolveram uma formulação de programa linear inteira para o problema de minimização de energia das redes $I P$ sobre $W D M$. No modelo proposto o modo de suspensão de equipamentos é ativado para dispositivos de proteção, considerando o $Q o P$. Apesar dos autores tratarem $Q o P$ em redes ópticas, eles não consideram $S D M-E O N$ e enfatizam a redução do consumo energético, diferentemente deste trabalho, que dedica-se em garantir proteção e maior taxa de aceitação para requisições de alta prioridade. Um algoritmo eficiente para provisionamento de acordo de nível de serviço (SLA - Service Level Agreement) com QoS aprimorada para EONs foi proposto em [Agrawal et al. 2017]. Nele, os autores consideram uma rede óptica que opera com serviços de diferentes requisitos, e propõe uma nova solução de roteamento 
e alocação de recursos que visa aumentar a eficiência da rede. Para isso são levados em consideração vários aspectos das conexões, dentre elas a proteção e a demanda por largura de banda. Porém, o trabalho não leva em consideração a aplicação em SDM-EON.

A proteção em redes ópticas elásticas empregando multiplexação por divisão espacial foi considerada em [Tan et al. 2016]. Os autores introduziram uma estratégia de provisionamento com ciência de diafonia através de métodos de proteção de caminho dedicado. $\mathrm{O}$ algoritmo proposto foi dividido em computação de roteamento e alocação de núcleo e espectro. Um algoritmo K-Shortest-Path $(K S P)$ foi empregado para encontrar caminhos primários e de backup emparelhados. Apesar de os autores tratarem proteção, eles não consideram $Q o P$, ignorando a relação de prioridades entre requisições. Um mecanismo de proteção e roteamento híbrido utilizando mono-caminho e multicaminhos em redes ópticas elásticas com multiplexação por divisão espacial foi proposto em [Oliveira and da Fonseca 2019]. O mecanismo proposto trata de proteção em SDM$E O N$, no entanto, desconsidera as classes de serviço de tráfego, utilizando o princípio de proteção para todos os fluxos. Tal metodologia resulta, muitas vezes, na aceitação de requisições com baixa prioridade e o bloqueio de requisições com alta prioridade.

Com base na análise do estado da arte é possível concluir que ainda há uma pequena quantidade de trabalhos relacionados a proteção em SDM-EON. Além disso, dos trabalhos que consideram a diferenciação dos serviços em classes, nenhum considera o uso da multiplexação espacial, o que é um problema, observando a enorme capacidade de comutação dessas redes, frente à tradicional WDM ou mesmo à EON.

\section{Algoritmo QoP-NOODLES}

Esta seção apresenta um novo algoritmo para roteamento e alocação de recursos em SDMEON considerando a proteção de caminho dos fluxos, ciente da prioridade atribuída à classe de serviço de cada requisição.

\subsection{Visão Geral da Rede}

A rede considerada na simulação utiliza enlaces bidirecionais com fibras ópticas de 7 núcleos, dispostos de forma hexagonal, cada núcleo contém um conjunto de 320 slots com capacidade de $12,5 \mathrm{GHz}$ cada. Em cada rota estabelecida, é aplicada a modulação fixa $B P S K$, o que significa a transferência de um bit por símbolo. A arquitetura utilizada para os nós da rede consiste no uso de multiplexadores ópticos add/drop reconfiguráveis, que permitem a adição, bloqueio, remoção e redirecionamento de feixes de luz de forma flexível, sensível à taxa de transmissão requisitada. Além disso, foi levado em consideração o emprego de uma rede óptica totalmente transparente, que evita conversões opto-elétricas e, portanto, que deve obedecer às restrições de continuidade e contiguidade de espectro e núcleo durante o estabelecimento dos caminhos ópticos.

Para a resiliência da rede, foi adotada a distinção das requisições em diferentes níveis de prioridade. Com esta abordagem, objetiva-se garantir que os fluxos com maior relevância tenham igual grau de $Q o P$, além de rápida recuperação durante situações desastrosas. Para tal, foi implementado um esquema de proteção dedicada de caminho óptico. Neste esquema, as conexões são divididas em 3 classes de serviço, cada uma possuindo diferente qualidade de proteção, da seguinte forma:

- Classe 1: fluxos desta classe possuem maior prioridade frente aos outros, sendo obrigados a possuir caminho de proteção e, portanto, possuem o maior $Q o P$. 
- Classe 2: nesta classe os fluxos possuem QoP média. Sendo assim, o algoritmo faz o maior esforço para prover um caminho de proteção para estes fluxos, porém, caso não consiga, ainda assim estabelece a conexão.

- Classe 3: esta é a classe que possui o menor $Q o P$. Os fluxos nela não exigem caminho de proteção, sendo perdidos em caso de falhas na rede que afetem seu caminho primário.

\subsection{Detalhes do QoP-NOODLES}

$\mathrm{O}$ algoritmo diferenciação de QoP para roteameNto, prOteção, alOcação De núcLeo e ESpectro (QoP-NOODLES), proposto nesta subseção, tem como objetivo elevar a taxa de aceitação e confiabilidade de fluxos com alto nível de relevância. Seu diferencial está na divisão do tráfego em diferentes graus de $Q o P$, elevando a eficiência na utilização de recursos ópticos. A melhor utilização de recursos é resultado da economia quanto à reserva de caminhos extras para backup, frente à política de proteção tradicional, que se fundamenta na tentativa de proteção de todos os fluxos, acentuando a curva de escassez dos recursos da rede. O algoritmo QoP-NOODLES será apresentado em três partes. No Algoritmo 1, dado o fluxo, são tratados o seu nível de proteção e a alocação de recursos a serem utilizados, ou o bloqueio do fluxo, se conveniente. No Algoritmo 2 (Algoritmo Seleção de Caminho Óptico - SCO) é tratada a escolha de rotas para o fluxo, selecionando o menor caminho óptico livre ou, caso necessária, a chamada para o Algoritmo 3 (Algoritmo Liberação de Recursos - LR), responsável pela liberação de caminho óptico de menor prioridade para uma nova alocação por um fluxo de maior prioridade.

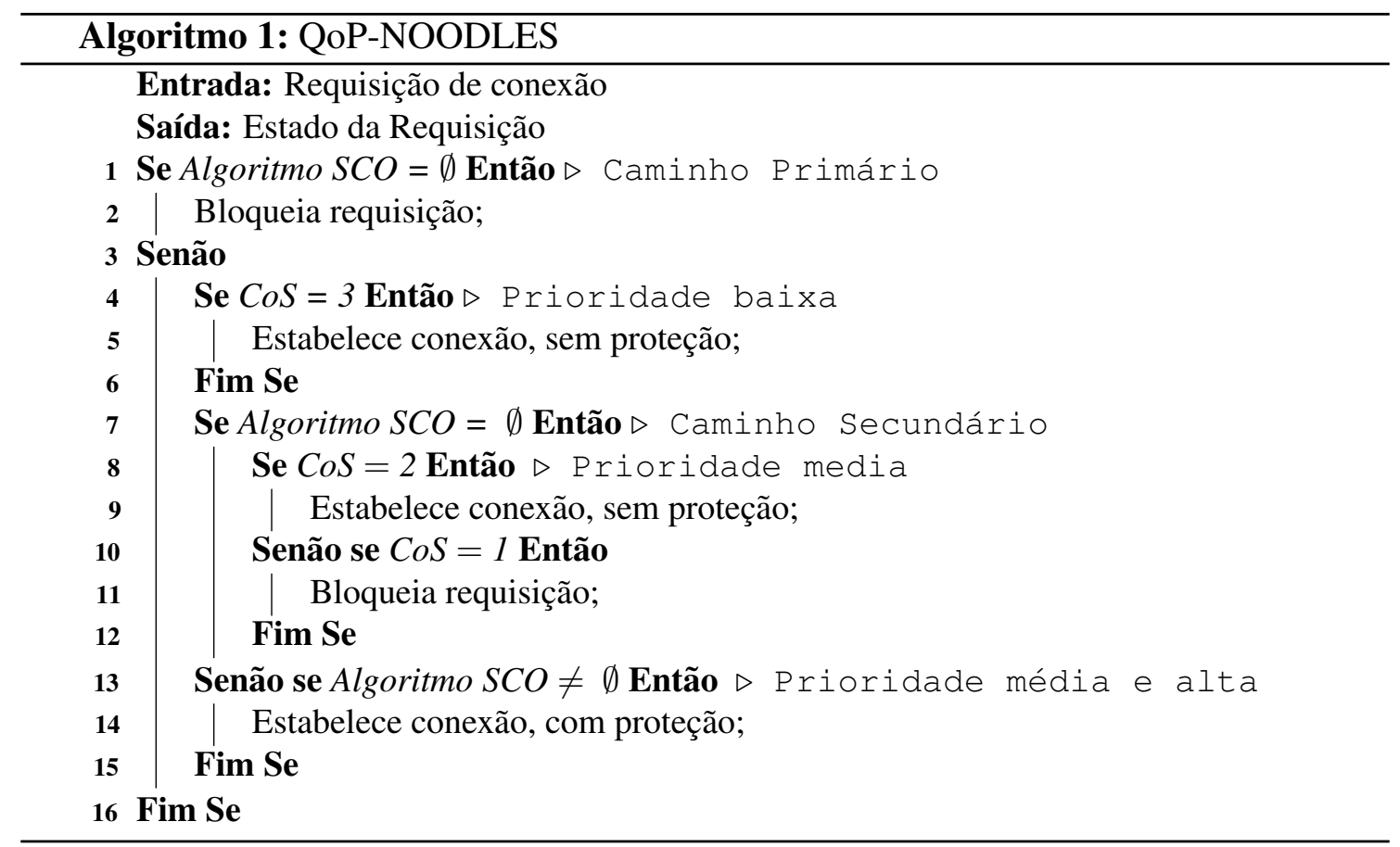

O Algoritmo QoP-NOODLES (Algoritmo 1) apresenta uma visão geral da lógica de alocação de recursos. Na Linha 1, o caminho primário é buscado, para isso o Algoritmo 2 (SCO) é utilizado. Caso o Algoritmo 2 não encontre nenhum caminho possível (retorno vazio), a requisição é bloqueada (Linha 2). Caso contrário, a proteção será tratada de acordo com a prioridade da requisição. A Linha 4 trata de requisições com baixa prioridade, e só será verdadeira para a classe 3 (conforme apresentado na seção 3.1). Quando verdadeira, a conexão é estabelecida sem proteção (Linha 5). Caso a requisição tenha prioridade média ou alta, o Algoritmo SCO é novamente cha- 
mado (Linha 7), porém agora em busca de um caminho de proteção. Vale ressaltar que nesta segunda chamada o Algoritmo 2 busca por caminhos disjuntos ao primário, excluindo a possibilidade de uma mesma falha inutilizar tanto o caminho principal quanto o de proteção. Se o retorno for nulo, significando que nenhum caminho possível foi encontrado, e a prioridade da requisição for média (Linha 8), então a conexão é estabelecida sem proteção (Linha 9). Ainda, se o retorno for nulo mas o $C o S$ da requisição for igual a 1 (prioridade alta), então esta é bloqueado (Linha 11). No entanto, se o retorno do SCO não for nulo (Linha 13), então a requisição é aceita com proteção dedicada (Linha 14).

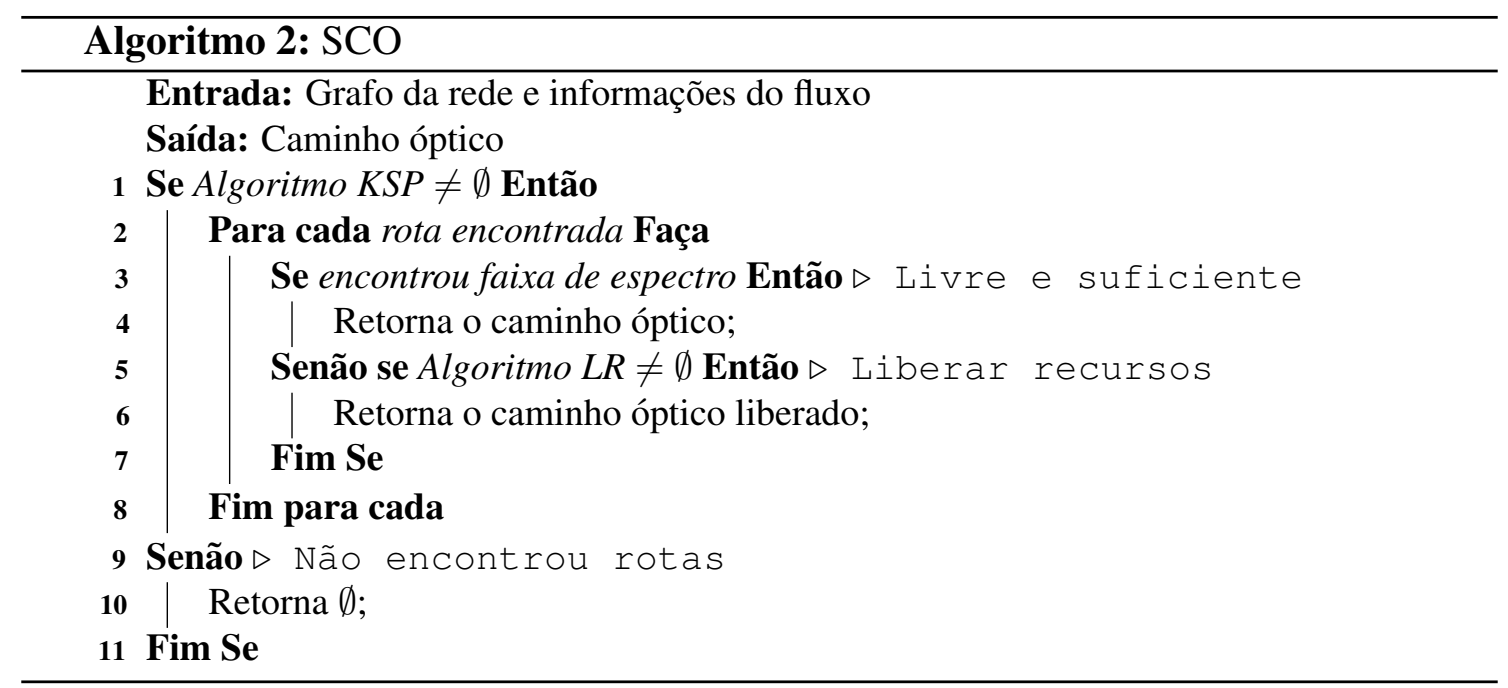

Em seguida, no Algoritmo SCO (Algoritmo 2) são expostas as etapas para a seleção de caminhos ópticos. O algoritmo recebe como entrada as informações da requisição e as informações da rede através de um grafo. Dentre as informações da requisição, pode-se citar a largura de banda requisitada, os nós fonte e destino da conexão e a sua classe de serviço. Como retorno para o Algoritmo 1, o SCO envia um caminho óptico, que consiste no conjunto de enlaces e slots a serem utilizados no transporte dos dado, ou um retorno nulo, caso não encontre caminho óptico possível de ser alocado. Na Linha 1, o algoritmo procura $K$ rotas possíveis entre um nó de origem e um nó de destino, através do algoritmo de [Yen 1971]. Vale ressaltar que, conforme o valor atribuído a $K$, o algoritmo $K S P$ pode retornar até 50 menores rotas entre os nós de origem e destino da conexão, para serem testados em relação à disponibilidade de banda. A utilização de múltiplos caminhos, visa aumentar ainda mais a taxa de aceitação dos fluxos.

Ainda sobre o Algoritmo SCO, a Linha 1 é a responsável pela análise quanto ao resultado do algoritmo KSP. Se esse for nulo (Linha 9), então o SCO é finalizado com um retorno vazio ao QoP-NOODLES (Linha 10). Na Linha 2, para cada uma das rotas encontradas é procurada uma porção do espectro contígua, contínua e livre o suficiente para atender os requisitos da requisição sob roteamento (Linha 3). Para isso é utilizada uma política Firt-Fit, que se baseia no mapeamento do espectro em ordem crescente. Sendo assim, a primeira faixa de espectro livre encontrada será a retornada. Portanto, caso seja encontrada largura de banda suficiente nesta rota, o SCO retornará o caminho óptico (Linha 4). Caso não seja encontrada nenhuma porção de espectro que atenda às restrições já citadas, então o Algoritmo 3 (Algoritmo LR) é chamado, para que este tente liberar um caminho óptico, baseando-se na rota e informações do fluxo, passadas como parâmetro (Linha 5). Por fim, caso o Algoritmo LR obtenha sucesso na liberação de recursos, o SCO retornará ao QoP-NOODLES o caminho óptico liberado (Linha 6).

O Algoritmo LR (Algoritmo 3) é responsável pela tentativa de liberação de recursos ópticos ocupados por requisições de menor prioridade, com o objetivo de dar espaço para nova alocação por requisições de maior prioridade. Para isso, ele recebe as informações da requisição 
e da rota sob avaliação. Como retorno ao Algoritmo SCO, o Algoritmo LR envia um caminho óptico, caso consiga uma liberação bem-sucedida, caso contrário devolverá um retorno vazio. Sua lógica se inicia na análise de cada índice de núcleo das fibras (Linha 1), buscando por porções de espectro que atendam a demanda do fluxo sob roteamento, além das restrições de continuidade e contiguidade de slots (Linha 2). Essa busca consiste na visita de cada slot do núcleo, reconhecendo quais dos enlaces da rota estão ocupados no slot e núcleo visitados.

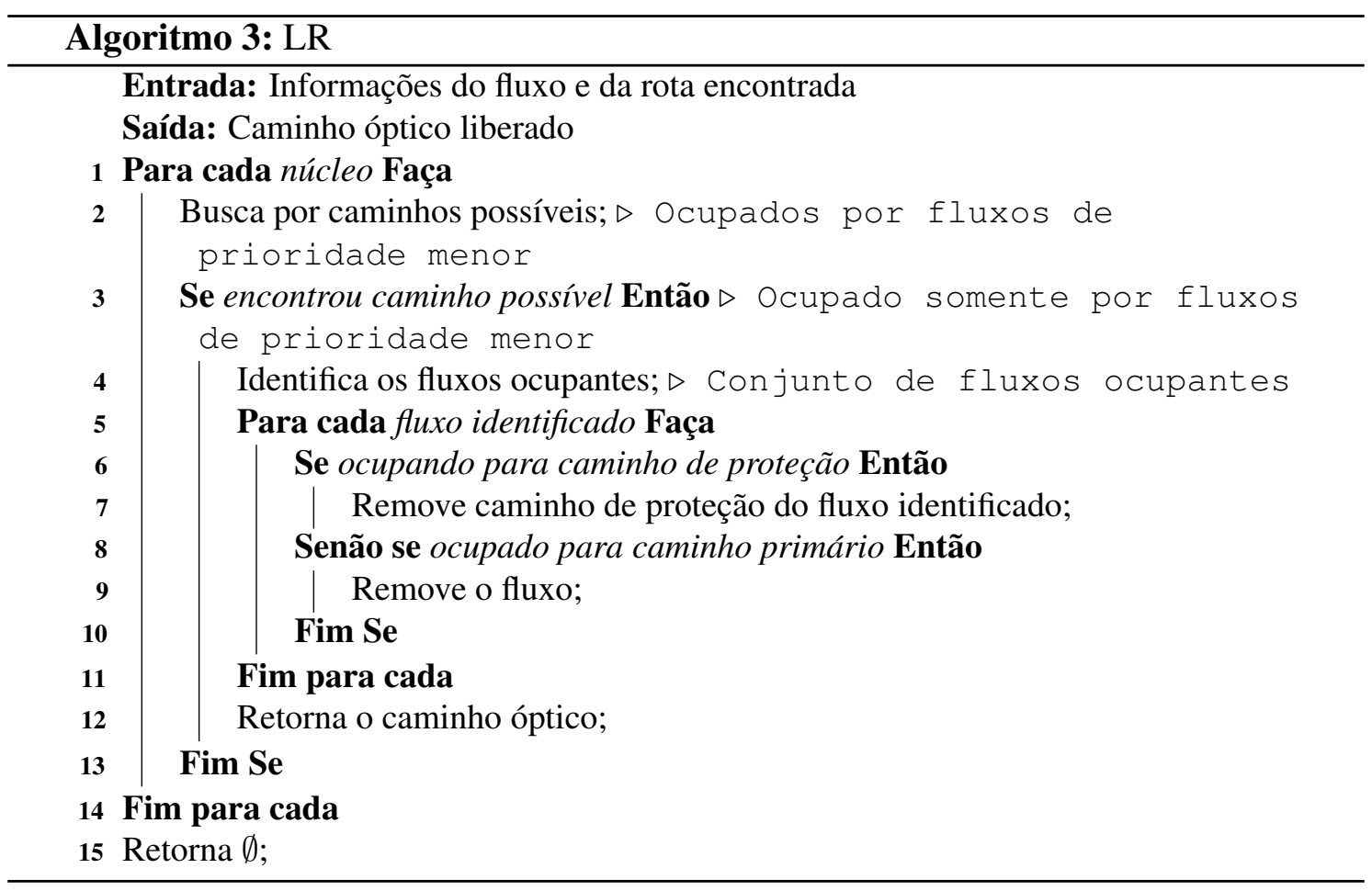

Nesta etapa, o Algoritmo LR busca pelos fluxos ocupados e avalia: se a remoção desses fluxos encontrados é admissível ( $\operatorname{CoS}$ maior); se a faixa de espectro ocupada, caso liberada, atenderá a demanda requisitada; e se é possível a combinação entre faixas de espectro ocupadas por fluxos de menor prioridade e faixas de espectro livres, contíguas e contínuas, para atender o fluxo com maior prioridade. Caso um caminho possível seja encontrado (Linha 3), então são identificados os fluxos a serem removidos (Linha 4). Na Linha 5, para cada um dos fluxos identificados é avaliado se a porção do espectro que será liberada está ocupada por um caminho de backup (Linha 6) ou primário (Linha 8). No caso de estar alocado para caminho de backup (Linha 6), apenas este caminho é removido, mantendo o fluxo identificado ainda ativo através de seu caminho primário. No entanto, caso a faixa de espectro solicitada esteja ocupada por um caminho primário (Linha 8), então o fluxo identificado é interrompido e seus recursos são liberados. Por fim, o caminho óptico liberado é retornado na Linha 12.

\section{Avaliação de desempenho}

Esta seção apresenta o ambiente de simulação, bem como os parâmetros e métricas utilizados para avaliar o desempenho do Algoritmo QoP-NOODLES.

\subsection{Descrição do cenário e metodologia}

Para a avaliação de desempenho, foram escolhidas duas topologias reais: a USA, Figura 1(a) com 24 nós e 43 enlaces e a NSF, Figura 1(b), que possui 14 nós e 25 enlaces. Foram executadas 100.000 requisições para cada simulação, com diferentes requisitos de largura de banda, variando em 25/50/125/200/500/750/1000 Gbps. Para a geração de tráfego foi utilizado o processo de 
Poisson, trabalhando com uma distribuição uniforme entre todos os pares de nós. Além disso, foi aplicada uma variação na carga de trabalho da rede, entre 25 e 400 erlangs $^{2}$, com diferença de 25 erlangs entre cada simulação. O Algoritmo QoP-NOODLES proposto foi avaliado através do simulador FlexGridSim [Moura and Drummond ]. Foram feitas algumas modificações em seu código-fonte para que fosse possível trabalhar com vários graus de $Q o P$.

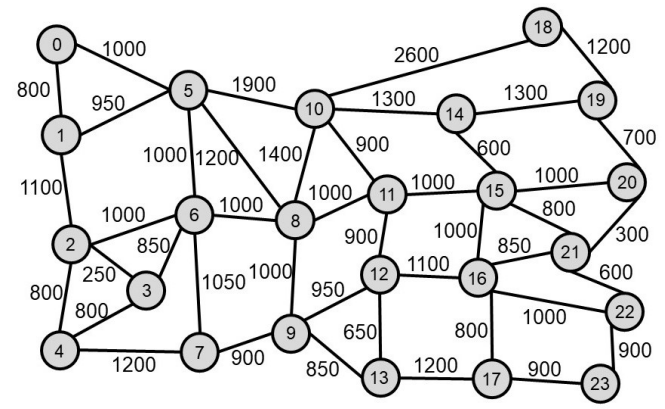

(a) Topologia USA

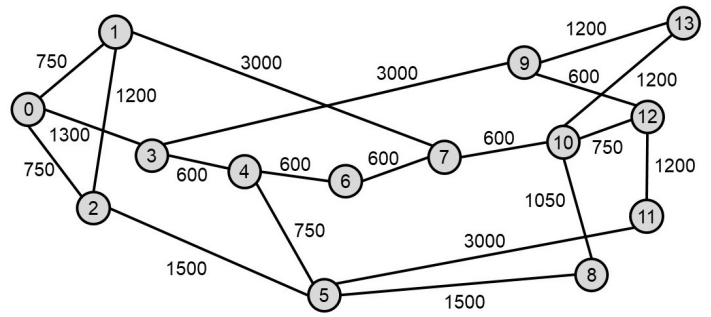

(b) Topologia NSF

Figura 1. Topologias

As métricas aqui avaliadas são: a probabilidade de bloqueio, que trata da relação entre a banda bloqueada e a banda total solicitada durante a simulação; a probabilidade de bloqueio para cada uma das 3 classes de serviço, que trata da relação entre a banda bloqueada para um $\operatorname{CoS} i$ e a banda total bloqueada durante a simulação; a razão de remoção de fluxos por $C o S$, que equivale à relação entre o número de fluxos removidos de um $\operatorname{CoS} i$ pelo número total de fluxos ativos para o mesmo $C o S$; a eficiência energética de cada um dos algoritmos, que corresponde à relação entre a quantidade de dados transportados pela infraestrutura da rede (em Mbits) pela quantidade de energia gasta pela mesma infraestrutura (em Joules).

\subsection{Resultados}

Nas figuras, as curvas com o rótulo "QoP-NOODLES" mostram o desempenho do Algoritmo QoP-NOODLES, proposto neste artigo. As curvas rotuladas com "MSP" mostram os resultados para um algoritmo genérico, denominado MSP, que representa uma abordagem tradicional de RSCA. O Algoritmo MSP se baseia apenas na busca por $K$ menores rotas e verificação da disponibilidade de banda para cada um deles. Se este encontrar caminho óptico disponível, o aloca, caso contrário, bloqueia a requisição. Além disso, vale ressaltar que o Algoritmo MSP não leva em consideração nenhuma outra variável, assim como a diferenciação em classes de serviço e a proteção dos caminhos ópticos. De outra forma, as curvas com o rótulo "MSP-P" demonstram o comportamento do Algoritmo MSP, já citado, mas com o incremento de uma política de proteção dedicada 1:1, seguindo a metodologia tradicional, sem distinção de tratamento para as diferentes classes de serviço. As curvas rotuladas com "CaP-DPP" [Tan et al. 2016] usam uma estratégia de provisionamento de proteção através de caminhos dedicados.

A Figura 2 mostra a probabilidade de bloqueio para cada topologia considerada neste artigo. Para a topologia USA (Figura 2(a)) os Algoritmos MSP-P e CaP-DPP, que empregam esquemas de proteção, iniciam o bloqueio requisições a partir de 50 erlangs. Por outro lado, o Algoritmo MSP que não considera a proteção inicia o bloqueio de requisições apenas a partir de 100 erlangs, isto ocorre pois a não utilização de proteção reduz a utilização de recursos e consequentemente aumenta a probabilidade de perdas em caso de falhas na rede. Apesar do Algoritmo QoP-NOODLES utilizar proteção, ele inicia o bloqueio de requisições a partir de 125 erlangs e mantém-se abaixo

\footnotetext{
${ }^{2}$ Erlang $=$ taxa média de chegada $\times$ tempo médio de duração dos fluxos
} 


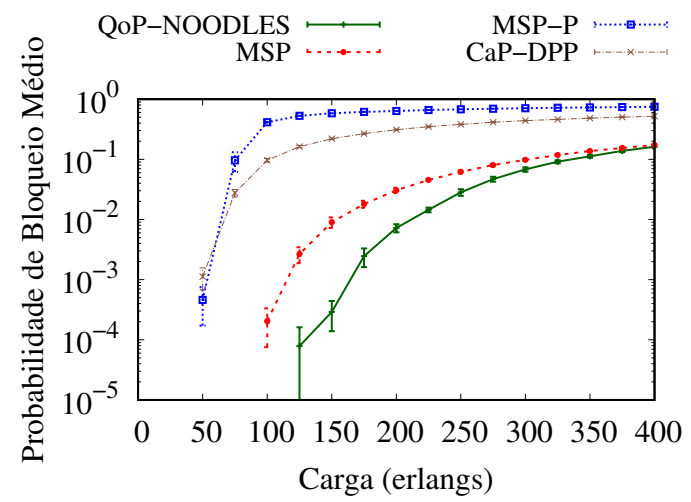

(a) Topologia USA

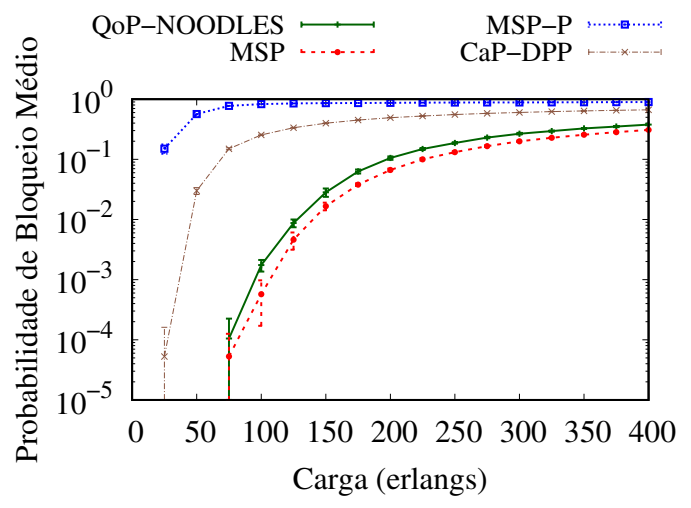

(b) Topologia NSF

Figura 2. Probabilidade de bloqueio

das outras curvas para todas as cargas simuladas. O baixo bloqueio de requisições no algoritmo QoP-NOODLES é consequência da melhor alocação de recursos.

Para a topologia NSF (Figura 2(b)), os Algoritmos MSP-P e CaP-DPP iniciam os bloqueios em 25 erlangs. Por outro lado, os Algoritmos QoP-NOODLES e MSP, começam a bloquear sob a carga de 75 erlangs. Pode-se notar que todos os algoritmos iniciaram os bloqueios em cargas menores, ao se comparar com o resultados para a topologia USA (Figura 2(a)). Isso é consequência do menor número de enlaces presentes na topologia NSF, o que resulta em menores possibilidades para o roteamento das requisições. Observa-se ainda que, quando comparado com o Algoritmo MSP, o QoP-NOODLES possui maiores taxas de bloqueio em todas as cargas simuladas. Isso se dá pelo fato de o Algoritmo MSP não exigir caminho de proteção durante o roteamento.

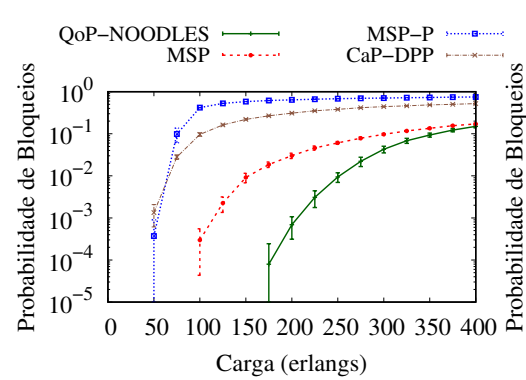

(a) $\operatorname{CoS} 1$

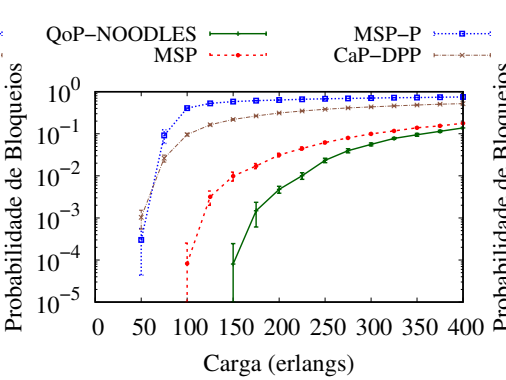

(b) $\operatorname{CoS} 2$

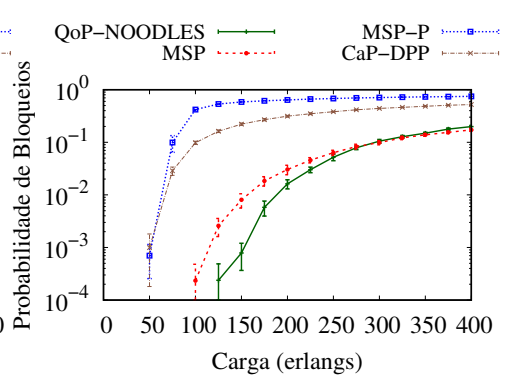

(c) $\operatorname{CoS} 3$

Figura 3. Probabilidade de bloqueio das diferentes CoS, para topologia USA

As Figuras 3 e 4 apresentam as relações de bloqueio para a classe de serviço. Para a topologia USA, na Figura 3, é notável a diferença entre a taxa de bloqueios do QoP-NOODLES, quando comparado com os demais algoritmos. Entretanto, pode-se notar também semelhanças entre as três figuras apresentadas, por exemplo, para todas as três classes de serviço (Figuras 3(a), 3(b) e 3(c)) os Algoritmos MSP-P e CaP-DPP iniciaram os bloqueios a partir da carga 50, e o MSP iniciou os bloqueios aos 100 erlangs. O bloqueio no Algoritmo QoP-NOODLES acontece somente sob maiores cargas nas Figuras 3(a), 3(b), iniciando em 175 e 150 erlangs, respectivamente, mantendo-se sempre abaixo das outras curvas. Contudo, a Figura 3(c), que representa a taxa de bloqueio dos fluxos com menor prioridade inicia o bloqueio em 125 erlangs, resultado próximo ao Algoritmo MSP, isto ocorre pois os fluxos de baixa prioridade podem ser removidos para possibilitar a locação de fluxos com maior prioridade. Observa-se também que as curvas 


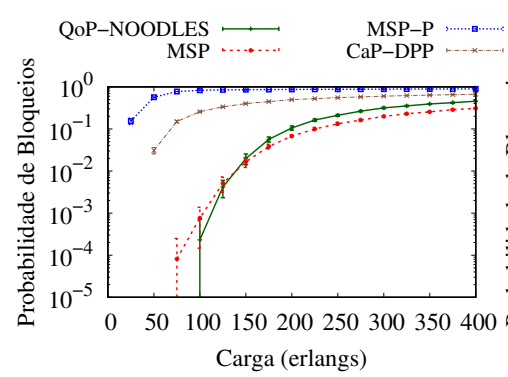

(a) $\operatorname{CoS} 1$

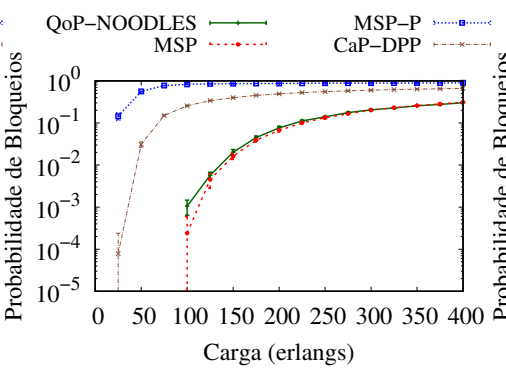

(b) $\operatorname{CoS} 2$

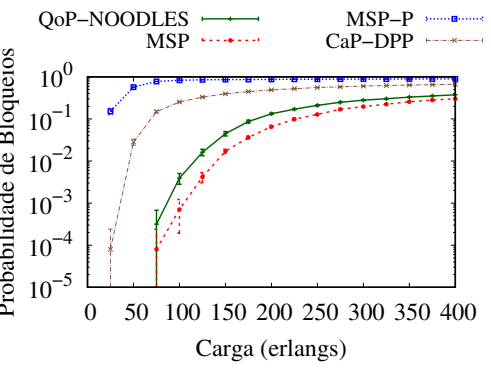

(c) $\operatorname{CoS} 3$

Figura 4. Probabilidade de bloqueio das diferentes CoS, para topologia NSF

com proteção dedicada, i.e., os Algoritmos MSP-P e CaP-DPP, produzem as maiores variações no intervalo entre 50 e 75 erlangs.

Para topologia NSF (Figura 4), percebe-se que as curvas referente aos Algoritmos MSP-P e CaP-DPP se mantêm acima das demais, assim como na Figura 3. Observou-se um aumento expressivo na probabilidade de bloqueios do Algoritmo QoP-NOODLES para todas as CoSs, além de esses bloqueios começarem a ocorrer em menores cargas, chegando à diferença de 75 erlangs, para a $\operatorname{CoS} 1$, quando comparada com os resultados da topologia USA. Isso acontece principalmente pelo fato de a topologia NSF possuir menor número de enlaces, diminuindo as possibilidades de estabelecimento de caminhos ópticos. Além disso mesmo com as remoções de fluxos com menor prioridade para o estabelecimento de conexões com prioridade maior, conforme a carga aumenta, a rede tende a carregar maior número de fluxos com $\operatorname{CoS}$ menor. Portanto, quanto maior o número de fluxos com alta prioridade ocupando os recursos da rede, menor é a probabilidade de remoção de fluxos com $\operatorname{CoS}$ maior, pois esses se tornam cada vez mais escassos.

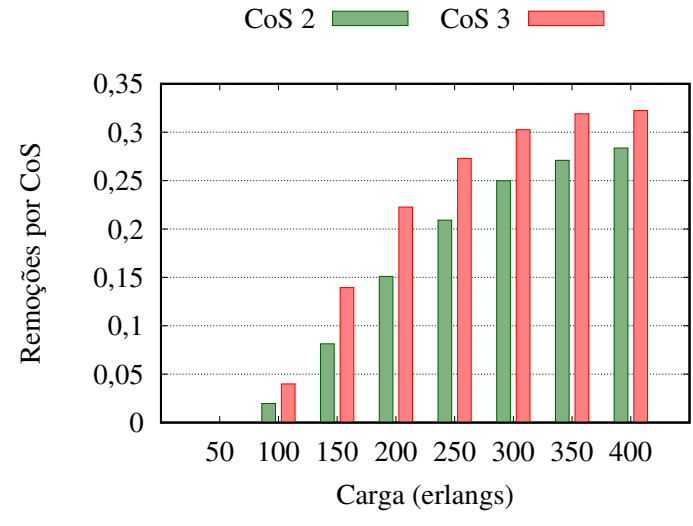

(a) Remoções USA

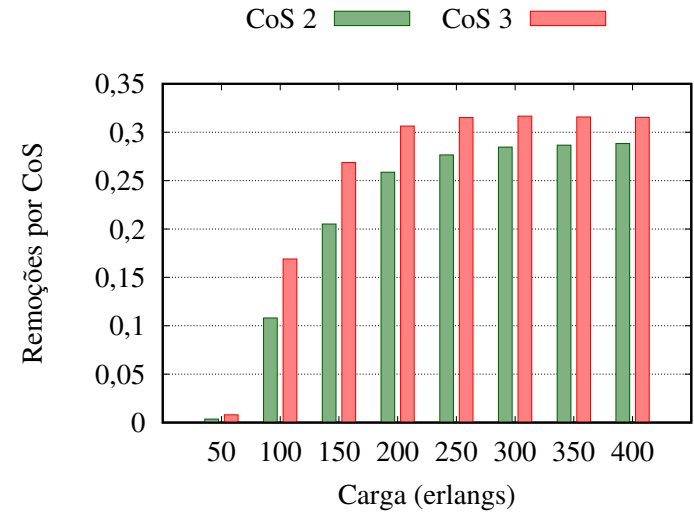

(b) Remoções NSF

Figura 5. Relação de remoção de fluxos por CoS para o QoP-NOODLES

Nas figuras 5(a) e 5(b) são mostradas as relações de remoção de fluxos para nova alocação por requisições de maior prioridade, das topologias USA e NSF, respectivamente. Nelas é possível notar para ambas as topologias, que o valor de remoções feitas pelo Algorítimo QoP-NOODLES convergem para 0,3, conforme a carga aumenta. Nota-se também que para a topologia NSF os valores de remoção se acentuam mais do que o os valores para a topologia USA. Isso fica evidente na diferença entre a razão de remoção de fluxos com $\operatorname{CoS} 3$, sob a carga de 100 erlangs, entre as duas topologias, que chega a mais de $10 \%$. Esse comportamento é devido ao menor número de enlaces presentes na topologia NSF, que faz com que os recursos da rede se tornem escassos sob menores cargas. 


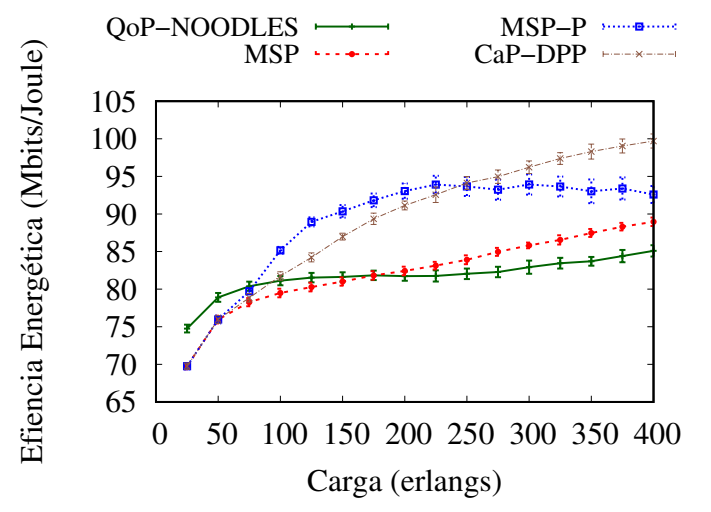

(a) Topologia USA

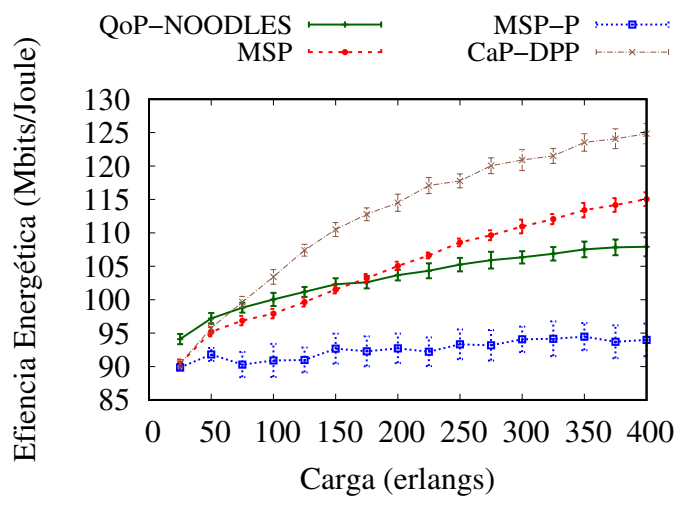

(b) Topologia NSF

Figura 6. Eficiência energética

A Figura 6 mostra a eficiência energética obtida nas topologias USA e NSF. Para topologia USA (Figura 6(a)), sob cargas altas, o Algoritmo CaP-DPP produz a maior eficiência energética, isso ocorre devido ao alto número de bloqueio de requisições, que faz com que apenas caminhos menores sejam estabelecidos. Além disso, percebe-se que a curva relativa ao Algoritmo MSP-P é a primeira que se estabiliza conforme a carga aumenta. Isso acontece pelo fato de o Algoritmo MSP-P trabalhar com uma política de proteção dedicada simples, só aceitando a requisição caso ambos os caminhos (primário e de proteção) sejam encontrados. Por conta disso, conforme a carga aumenta, os recursos ficam mais escassos. Ainda, é possível perceber que a curva que demonstra a eficiência energética produzida pelo Algoritmo QoP-NOODLES se mantém abaixo das demais. Isso pode ser explicado pelo fato de a rede sempre buscar manter maior número de fluxos de $\operatorname{CoS} 1$ ativos, que exigem caminhos de proteção para serem aceitos e, portanto, consumindo maior quantidade de recursos que, durante a não ocorrência de falhas, não transportam dados mas mantêm o consumo energético.

Para topologia NSF (Figura 6(b)), sob cargas maiores que 75 erlangs, o Algoritmo CaPDPP apresenta melhor eficiência energética que os demais algoritmos. Isso ocorre, pois, o maior bloqueio do algoritmo CaP-DPP, influencia no número de saltos dos caminhos, que consequentemente aumenta a eficiência energética. De outra forma, percebe-se que o Algoritmo MSP-P possui uma curva estável durante todo o gráfico, indicando baixo aproveitamento energético, gerado pela saturação dos recursos da rede. Esse comportamento, por outro lado, significa maior número de enlaces e nós solicitados, mas sem transporte de dados. Por fim, os Algoritmos QoP-NOODLES e MSP, apresentam comportamentos parecidos, quando comparados com a Figura 6(a), porém os valores em Mbits/Joule apresentados por eles demonstram maior eficiência energética em comparação com os dados correspondentes à topologia USA.

\section{Conclusões}

Neste artigo, foram avaliados o aumento da quantidade de tráfego através de SDM-EON bem como a heterogeneidade destes fluxos e importância da resiliência nestas redes. Além disso, realizou-se uma análise através de simulações para entender o comportamento da rede quanto à capacidade de estabelecimento de conexões para fluxos com diferentes classes de serviço, quando essa se encontra sobrecarregada. Foi proposto um novo algoritmo de proteção para SDM-EON, tendo em vista a grande capacidade de transmissão que estas podem oferecer, com o objetivo de garantir um melhor tratamento para os tráfegos de maior prioridade. $\mathrm{O}$ algoritmo proposto, obteve menor probabilidade de bloqueio, especialmente na probabilidade de bloqueios de requisições com maior prioridade, quando comparado com outros algoritmos da literatura. Além disso, pôde-se garantir maior QoP para fluxos de maior relevância, utilizando uma política de diferenciação de serviços, 
que garante maior eficiência na utilização dos recursos da rede. Como trabalhos futuros, pretendese considerar também a modulação adaptativa e diafonia gerada entre núcleos, em decibéis, tendo em vista maior aproveitamento do espectro e menor perda de dados durante o transporte.

\section{Referências}

Agrawal, A., Vyas, U., Bhatia, V., and Prakash, S. (2017). Sla-aware differentiated QoS in elastic optical networks. Optical Fiber Technology, 36:41-50.

Boutaba, R., Shahriar, N., and Fathi, S. (2017). Elastic optical networking for 5g transport. Journal of Network and Systems Management, 25(4):819-847.

Cisco, U. (2020). Cisco annual internet report (2018-2023) white paper. https://www . cisco.com/c/en/us/solutions/collateral/executive-perspectives/ annual-internet-report/white-paper-c11-741490.html. Accessed: 2020-04-02.

De La Torre Díez, I., Alonso, S. G., Hamrioui, S., López-Coronado, M., and Cruz, E. M. (2018). Systematic review about QoS and QoE in telemedicine and ehealth services and applications. Journal of medical systems, 42(10):182.

Hai, D. T. (2020). On the spectrum-efficiency of QoS-aware protection in elastic optical networks. Optik, 202:163563.

Hmaity, A., Musumeci, F., and Tornatore, M. (2018). Power reduction strategies with differentiated quality of protection in ip-over-wdm networks. Annals of Telecommunications, 73:81-94.

Laghari, A. A., He, H., Memon, K. A., Laghari, R. A., Halepoto, I. A., and Khan, A. (2019). Quality of experience $(\mathrm{QoE})$ in cloud gaming models: A review. Multiagent and Grid Systems, 15(3):289-304.

Moura, P. M. and Drummond, A. C. FlexGridSim: Flexible Grid Optical Network Simulator. http://www.lrc.ic.unicamp.br/FlexGridSim/.

Oliveira, H. M. N. S. and da Fonseca, N. L. S. (2019). Multipath routing, spectrum and core allocation in protected sdm elastic optical networks. In 2019 IEEE GLOBECOM, pages 1-6.

Santos, A. S., de Santi, J., and Figueiredo, G. B. (2018). Uma estratégia online para degradação de serviço com QoS proporcional em redes ó pticas elásticas. In Anais do XXXVI Simpósio Brasileiro de Redes de Computadores e Sistemas Distribuídos. SBC.

Tan, Y., Zhu, R., Yang, H., Zhao, Y., Zhang, J., Liu, Z., Qu, Q., and Zhou, Z. (2016). Crosstalkaware provisioning strategy with dedicated path protection for elastic multi-core fiber networks. In 2016 15th International Conference on Optical Communications and Networks (ICOCN), pages 1-3. IEEE.

Yen, J. Y. (1971). Finding the k shortest loopless paths in a network. Management Science, 17(11):712-716.

Zhang, J. and Mukheriee, B. (2004). A review of fault management in WDM mesh networks: basic concepts and research challenges. IEEE network, 18(2):41-48. 